\title{
Biomineralization of Hydroxyapatite Revealed by in situ Electron Microscopy
}

Xiaoyue Wang ${ }^{1}$, Jie Yang ${ }^{2}$, Carmen Andrei ${ }^{3}$, Leyla Soleymani ${ }^{2,4}$ and Kathryn Grandfield ${ }^{1,2}$

${ }^{1 .}$ Department of Materials Science and Engineering, McMaster University, Hamilton, Canada.

2. School of Biomedical Engineering, McMaster University, Hamilton, Canada.

3. The Canadian Centre for Electron Microscopy, McMaster University, Hamilton, Canada.

4. Department of Engineering Physics, McMaster University, Hamilton, Canada.

Hydroxyapatite (HA) is the principal mineral component of human bones and teeth. Within these mineralized tissues, HA distributes itself in a highly organized arrangement in a fibrous collagen matrix. Although many bone mineralization mechanism theories have been put forward based on in vitro models, many questions such as the formation of an amorphous calcium phosphate (ACP) intermediary, and the functions of non-collagenous proteins (NCPs) on crystals nucleation and subsequent growth, still remain due to the lack of real-time experimental evidence [1]. Liquid-phase transmission electron microscopy (LP-TEM), with the ability to record biomineralization process in situ with nanoscaled spatial resolution and sufficient temporal resolution, is a promising technique to unveil bone mineralization mechanisms. The formation of intermediate amorphous phases has been noted in situ for simpler mineralized systems, such as calcium carbonate [2]. However, understanding the mechanisms of more complex HA mineralization is critical for both pathological biomineralization research and biomimetic material synthesis related to humans.

In this work, an HA biomineralization solution was placed in a static liquid cell inside the TEM to observe HA particle nucleation and growth. A JEOL 2010F was operated at $200 \mathrm{keV}$ in combination with a commercially available liquid cell TEM holder (Poseidon 500, Protochips Inc., USA). A small amount $(0.2 \mu \mathrm{L})$ of HA biomineralization solution was sealed between stock microwell Poseidon Echips with SiN windows. The solution was made up of two parts: 1) a phosphate solution $(9.5 \mathrm{mM}$ $\mathrm{Na}_{2} \mathrm{HPO}_{4}$ in $125 \mathrm{mM} \mathrm{NaCl}, 50 \mathrm{mM}$ Tris, $\left.\mathrm{pH} 7.40\right)$, and 2) a calcium solution $\left(1.7 \mathrm{mM} \mathrm{CaCl}_{2}\right.$ in $125 \mathrm{mM}$ $\mathrm{NaCl}, 50 \mathrm{mM}$ Tris, $\mathrm{pH}$ 7.40) [3]. These two solutions were mixed and filtered through a $0.2 \mu \mathrm{m}$ acrodisc syringe filter right before sealing in the liquid TEM holder. The electron beam dose rate was estimated at $2.198 \times 10^{3} \mathrm{e} / \AA^{2} \cdot \mathrm{s}$. A thinner liquid region near the edge of the microwells was used for imaging. After the in situ experiment, the E-chips were removed from the liquid TEM hold and dried for further ex situ characterization via X-Ray Photoelectron Spectrometry (XPS) and electron energy loss spectroscopy (EELS).

In scanning-TEM bright-field imaging mode (BF-STEM), tiny particles with an average diameter of around $10 \mathrm{~nm}$ appeared after 2 minutes. With increasing time, more particles appeared and these had the ability to move and aggregate with each other to generate clusters instead of growing into bigger particles individually (Figure 1). These particles were confirmed as HA crystals via in situ selected area diffraction patterns in TEM mode, and also supported by complementary ex situ characterization with EELS (Figure 2) and XPS.

We present the first real-time imaging of HA nucleation and growth with in situ LP-TEM. This is the first step in understanding HA biomineralization in more complex organic-inorganic physiological solutions. This work lays the foundation for further investigation of HA nucleation in the presence of NCPs, and for the exploration of remineralization of extracellular matrices such as collagen in situ. 


\section{References:}

[1] M. J. Olszta, X. Cheng and S. S. Jee. Materials Science and Enginnering: Reports, 58 (2007), p.77.

[2] P. J. M. Smeets, K. R. Cho and R. G. E. Kempen. Nature Materials, 14 (2015), p. 394.

[3] A. J. Lausch, B. D. Quan and J. W. Miklas. Advanced Functional Materials, 23 (2013), p. 4906.

[4] The authors acknowledge funding from the Natural Sciences and Engineering Research Council of Canada (NSERC)

Discovery Grant program. Microscopy was performed at the Canadian Centre for Electron Microscopy at McMaster University, a facility supported by NSERC and other government agencies.

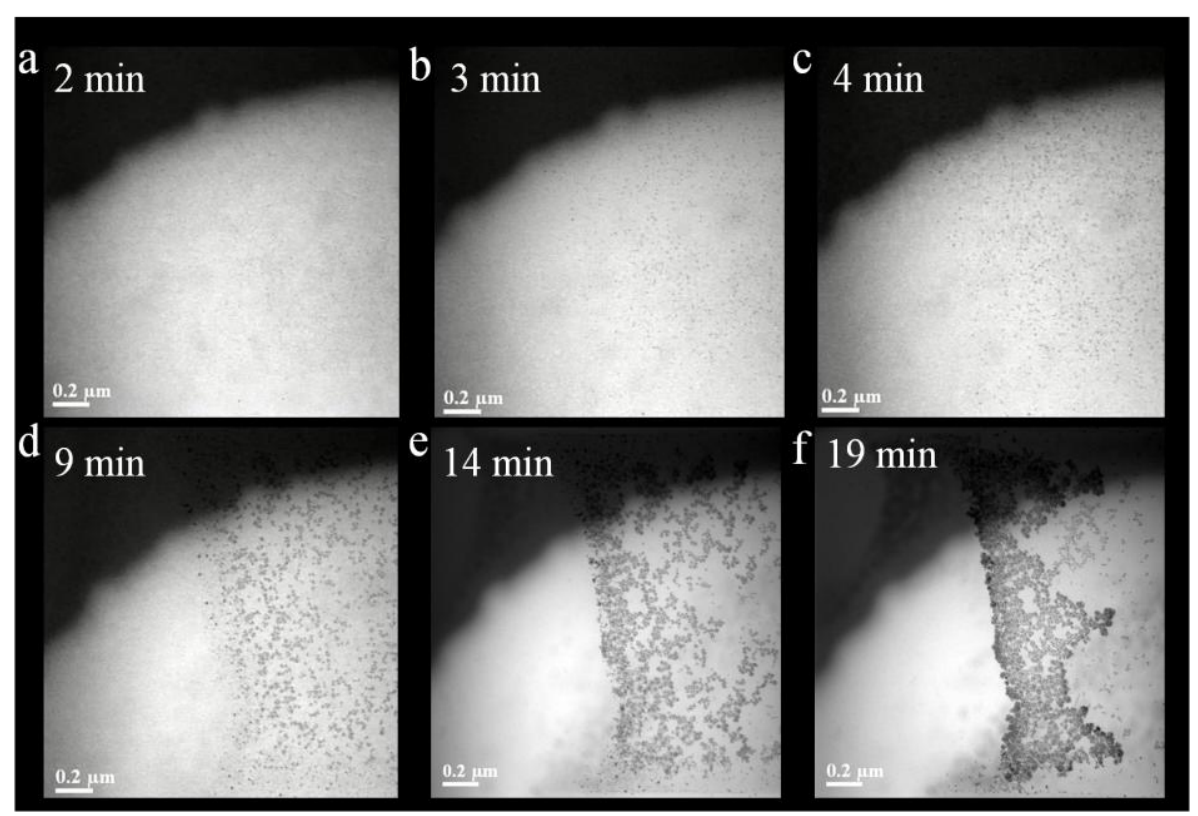

Figure 1. In situ BF-STEM images showing initial nucleation and growth of HA particles over 20 min.

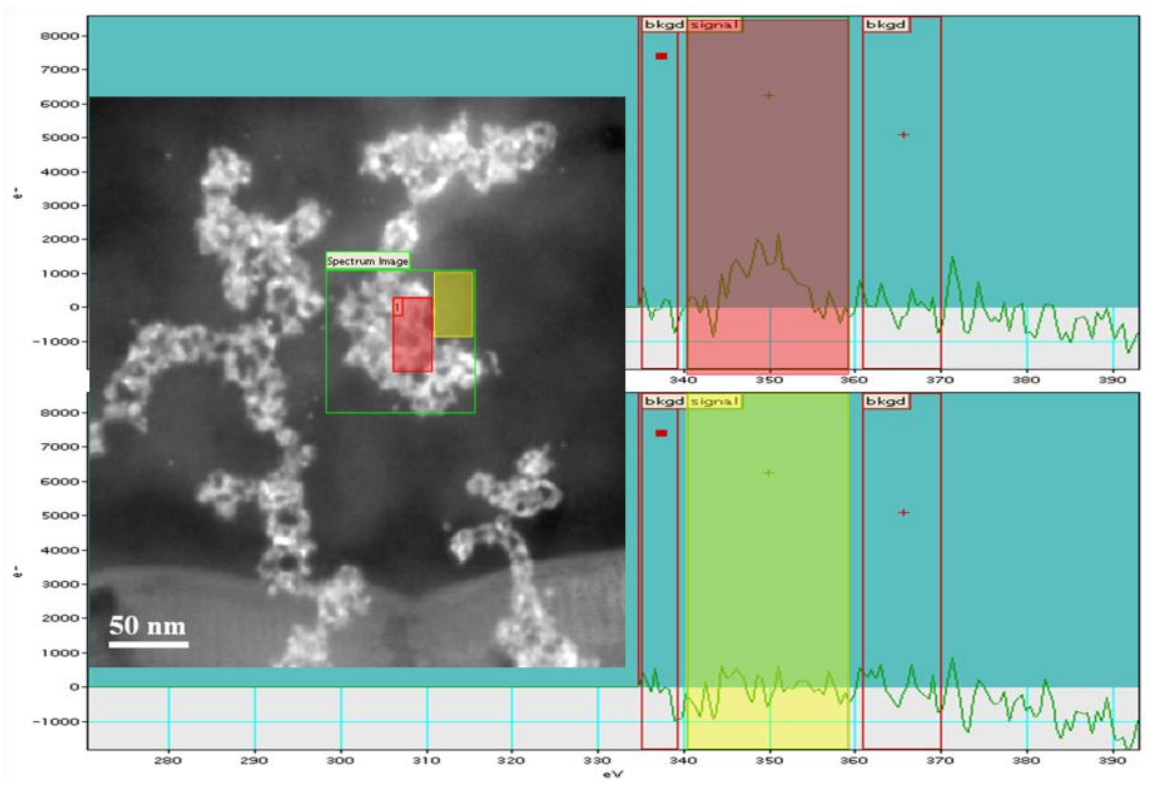

Figure 2. The mineralized product was characterized via EELS. The Ca edge was identified inside mineralized particles (red), and absent in the surrounding regions (yellow). Selected area electron diffraction, and XPS confirmed the chemical structure of these Ca-rich particles to be hydroxyapatite. 